\title{
Efficacy of combination therapy with zoledronic acid and cetuximab for unresectable rectal cancer with bone metastases: A case report
}

\author{
YOSHIHISA TOKUMARU, NOBUHISA MATSUHASHI, TAKAO TAKAHASHI, TOSHIYUKI TANAHASHI, \\ SATOSHI MATSUI, HISASHI IMAI, YOSHIHIRO TANAKA, KAZUYA YAMAGUCHI and KAZUHIRO YOSHIDA \\ Department of Surgical Oncology, Gifu University School of Medicine, Gifu, Gifu 501-1194, Japan
}

Received May 7, 2018; Accepted March 14, 2019

DOI: $10.3892 /$ mco.2019.1836

\begin{abstract}
The combination of cetuximab (CTX) and chemotherapy, such as FOLFOX or FOLFIRI, is currently the standard treatment for metastatic colorectal cancer (mCRC). Zoledronic acid (ZOL) is used in patients with bone metastasis. We herein report our experience with the case of a 58-year-old male patient with metastatic rectal cancer who was treated with ZOL + CTX as third-line therapy, and in whom this combination appeared to be effective. Although the patient developed bone metastasis and cardiac tamponade due to the recurrence of rectal cancer, he survived for approximately 10 months after the initiation of ZOL and CTX treatment.
\end{abstract}

\section{Introduction}

Colorectal cancer (CRC) was estimated to account for 881,000 deaths worldwide in 2018 and it remains one of the major causes of death globally (1). The incidence of distant metastasis is as high as $25 \%$ at initial diagnosis, and approximately half of the patients with CRC will develop metastatic disease (2).

Cetuximab (CTX; Erbitux, Merck KGaA, Darmstadt, Germany) is a monoclonal antibody that inhibits ligand binding and ligand-dependent downstream signaling by specifically targeting the epidermal growth factor receptor (EGFR) $(3,4)$. Accumulation of data from several clinical studies revealed

Correspondence to: Dr Nobuhisa Matsuhashi, Department of Surgical Oncology, Gifu University School of Medicine, 1-1 Yanagido, Gifu, Gifu 501-1194, Japan

E-mail: nobuhisa517@hotmail.com

Abbreviations: ADCC, antibody-dependent cytotoxicity; CRC, colorectal cancer; CT, computed tomography; CTX, cetuximab; EGFR, epidermal growth factor receptor; ERK, extracellular signal-regulated protein kinase; MAPK, mitogen-activated protein kinase; mCRC, metastatic CRC; ZOL, zoledronic acid

Key words: zoledronic acid, cetuximab, unresectable rectal cancer, bone metastases the efficacy of CTX combined with the standard therapy arm (FOLFOX/FOLFIRI) in treating patients with metastatic CRC (mCRC) with wild-type KRAS $(5,6)$. Therefore, in the clinical setting, the use of CTX is currently restricted to patients with wild-type mCRC.

Zoledronic acid (ZOL) is a member of the bisphosphonate molecular class and is used in the clinical setting to reduce skeletal-related events in patients with bone metastasis. Previous studies reported on the antitumor activity of ZOL against several human cancers, such as breast, prostate and colorectal cancers, in vitro or in vivo $(7,8)$.

We herein report the clinical course and computed tomography $(\mathrm{CT})$ imaging findings in a patient with $\mathrm{mCRC}$ treated with ZOL and CTX.

\section{Case report}

A 58-year-old male patient presented to Gifu University Hospital (Gifu, Japan) with abdominal pain, symptoms of colonic penetration due to stenosis caused by rectal cancer in January 2014, and initially underwent an ileostomy. Peritoneal dissemination was detected during the ileostomy procedure, and CTX + mFOLFOX was started to treat the unresectable wild-type KRAS CRC. Analysis of RAS and EGFR status performed by SRL, Inc. (Tokyo, Japan) revealed wild-type RAS and high expression of EGFR in this patient. The patient underwent 6 courses of CTX + mFOLFOX before undergoing low anterior resection and D3 lymphadenectomy as the second surgery. Since R0 resection could be performed, 6 courses of mFOLFOX were administered as postoperative chemotherapy, after which time the patient was observed. A follow-up CT scan 6 months after the second surgery revealed a metastasis to the thoracic spine that was treated with X-ray radiation therapy $20 \mathrm{~Gy} / 5 \mathrm{fr}$.

Approximately 9 months after the second surgery, the patient complained of gradual onset of fatigue and shortness of breath; a chest CT scan revealed pericardial effusion and cardiac tamponade, which were managed by pericardial drainage (Fig. 1).

At 1 year after the second surgery, the patient began to have difficulty walking, which was caused by the thoracic spine metastasis and compression of the spinal cord. At that 
time, combination therapy with CTX and ZOL (CTX: $400 \mathrm{mg}$ weekly, ZOL: $4 \mathrm{mg} /$ body tri-weekly) was initiated. There were several reasons for selecting CTX and ZOL in this patient: First, the idea of using irinotecan (CPT-11) was abandoned, as the patient had cardiac and pleural effusion. Second, we hypothesized that the tumor was refractory to oxaliplatin, as recurrence occurred within 3 months after chemotherapy. An alternative treatment for this patient would be the administration of regorafenib or TAS-102; however, these drugs are associated with increased risks in patients with renal dysfunction, such as in the present case.

Following treatment with CTX and ZOL, the size of the thoracic and lung metastasis was found to be decreased on CT imaging $\sim 5$ months following the initiation of the combination therapy. In parallel with these findings, the levels of the tumor markers carcinoembryonic antigen (normal $<5.0 \mathrm{ng} / \mathrm{ml}$ ) and carbohydrate antigen 19-9 (normal $<37.0 \mathrm{U} / \mathrm{ml}$ ) also decreased from 15.1 to 8.6 and 266.8 to 38.2 respectively (Figs. 2 and 3). However, the disease gradually progressed 3 months after the initiation of the ZOL + CTX combination therapy. In total, the patient was administered 28 courses of CTX and 8 courses of ZOL, but eventually succumbed to the disease 2 years and 3 months after the first surgery.

\section{Discussion}

Following its approval in 2004, bevacizumab, a humanized recombinant monoclonal antibody against vascular endothelial growth factor-A, became the first biological therapy for the initial treatment of mCRC. Bevacizumab achieved improvements in progression-free and overall when combined with chemotherapy, such as FOLFIRI and FOLFOX, in several studies $(9,10)$. In the same year, a randomized trial of CTX alone or in combination with CPT-11 exhibited clinical effectiveness in CPT-11-refractory CRC, confirming the results of phase 2 studies of third-line therapy. CPT-11 and combination therapy achieved markedly higher response rates compared with CTX monotherapy alone. However, CTX monotherapy was effective compared with placebo and was associated with only mild toxicity, including skin toxicity; thus, it may be a viable option for patients who are not considered candidates for further treatment or who choose not to receive such combination therapy (11). In addition, in 2006, CTX, which is a monoclonal antibody against EGFR, was introduced as treatment for CRC and these drugs are currently considered to largely contribute to the better prognosis of patients with CRC.

Molecules of the EGFR family constitute a signaling pathway that plays an important role in intracellular reactions. Three major pathways are involved in EGFR-mediated signaling. The first pathway is that of the mitogen-activated protein kinase (MAPK) cascades. EGFR tyrosine autophosphorylation activates Ras, which leads to a multistep phosphorylation event and subsequently activates MAPKs and extracellular signal-regulated protein kinase (ERK)1 and ERK2. These kinases regulate a large number of molecules that are involved in cell transformation, proliferation and survival (12). Another important pathway secondary to the EGFR activation is phosphatidylinositol-3-kinase and the downstream protein serine/threonine kinase Akt. Akt transduces intracellular signals linked to cell survival,

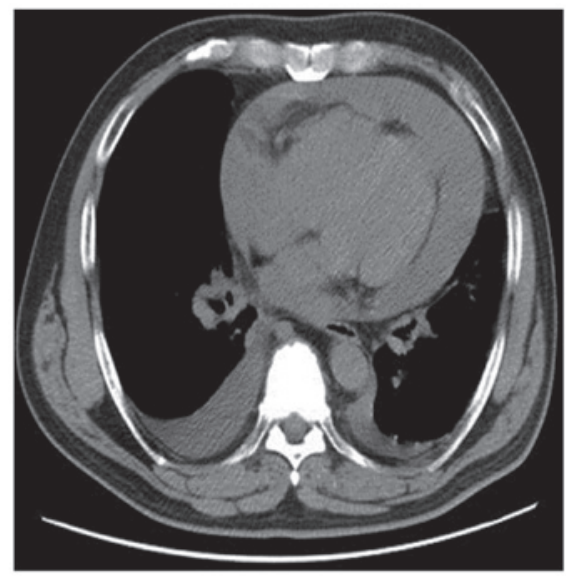

Figure 1. Computed tomography scan showing the pericardial effusion.

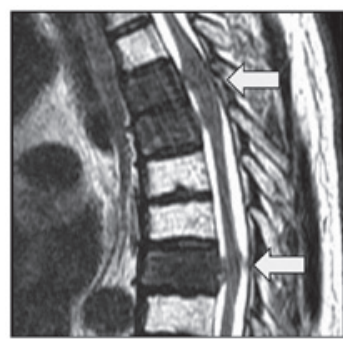

Prior to treatment of ZOL+CTX

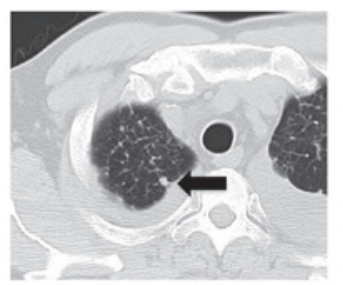

Figure 2. Comparison of computed tomography (CT) scan before and after the administration of ZOL + CTX. The CT images show a reduction in the size of the metastatic lesions (arrows). Top row, bone metastasis; bottom row, lung metastasis. ZOL, zoledronic acid; CTX, cetuximab.

proliferation and motility (13). The third pathway is represented by Jak2/STAT3. EGF, the mitogenic hormone that activates EGFR, plays an important role in regulating the proliferation and differentiation of normal and neoplastic cells in vitro and in vivo.

As the newest nitrogen-containing bisphosphonate, the suppression of bone resorption of ZOL increases by 2,000 times in comparison with that of flexor chloride sodium phosphate, and it is 200 times that of pamidronate, which is widely used to prevent and treat bone metastases from solid tumors, and to delay the development of cancer-related bone damage. Clinical experience has proven that ZOL does not only prevent bone diseases, but also affects cancer activity by inhibiting the proliferation and reducing the activity of cancer cellos (14). The efficacy of combination therapy with ZOL and CTX on CRC cells was previously reported in a preclinical study (8). The administration of ZOL carries the risk of osteonecrosis of jaw. However, this side effect did not occur for this patient.

There are two mechanisms underlying the antitumor effect of the ZOL and CTX combination therapy in our patient: One 


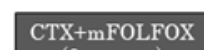
(6 courses)
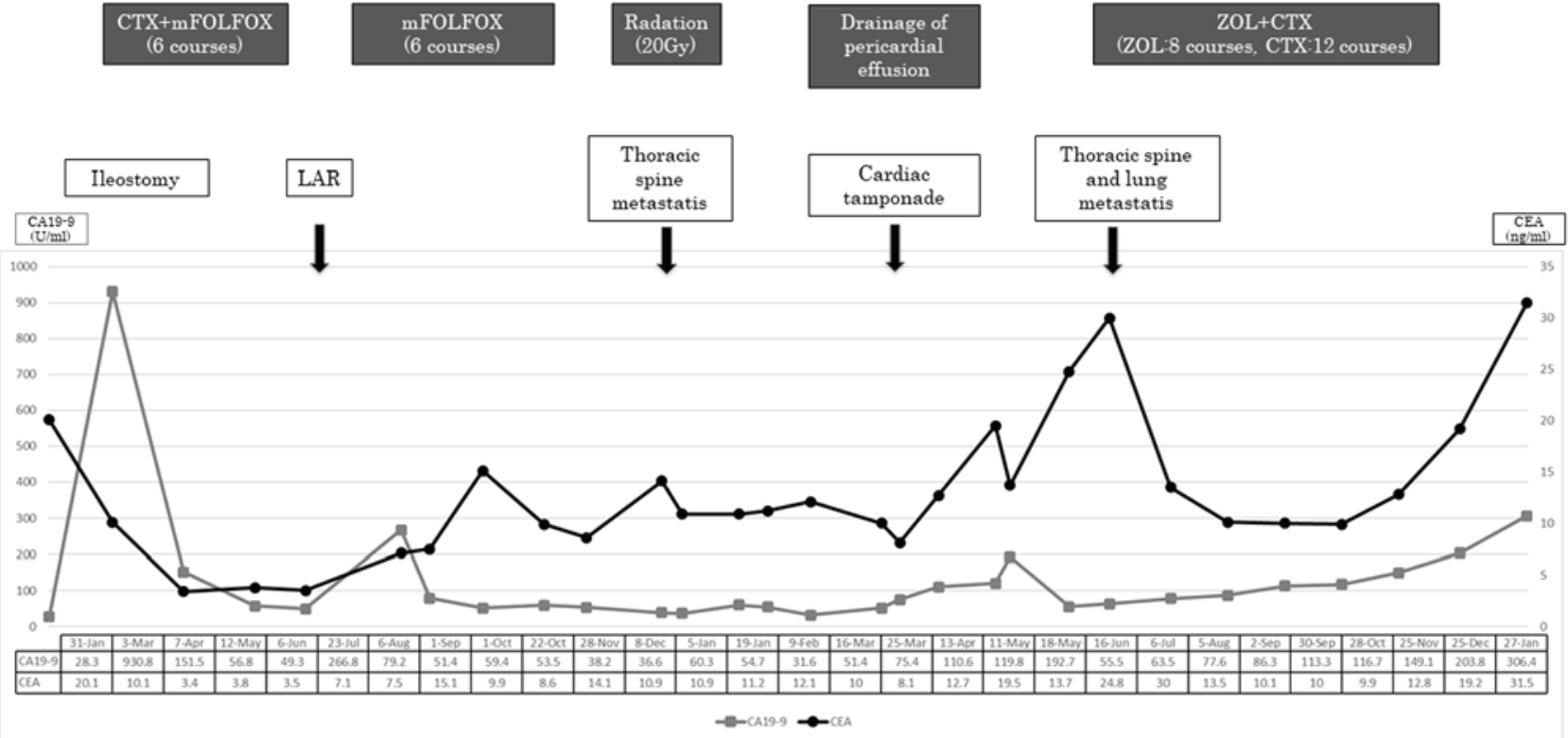

Figure 3. Tumor marker levels over the course of treatment. CEA, carcinoembryonic antigen; CA19-9, carbohydrate antigen 19-9; ZOL, zoledronic acid; CTX, cetuximab; LAR, lower anterior resection.

mechanism includes the inhibition of RAS signaling by both agents. In KRAS wild-type tumors, CTX inhibits the intracellular signaling cascades, such as the RAS signaling pathways that promote cell growth, by competing with the ligand for binding to the EGFR receptor $(3,15)$. In a preclinical study, ZOL exerted an antitumor effect on CRC by inhibiting prenylation of RAS (8). The other mechanism is the antitumor activity occurring via targeting pathways unrelated to EGFR, such as antibody-dependent cytotoxicity (ADCC) (16). A previous report suggested that ZOL enhances ADCC by increasing the number of $\delta \gamma T$ cells (17). This evidence suggests that combination therapy with ZOL and CTX may synergistically enhance ADCC activity and exert an antitumor effect.

In our patient, radiotherapy alone was selected after the recurrence to the thoracic spine, as there are currently no reports confirming the effectiveness of an anti-EGFR agent against the bone metastases of CRC in the English literature; furthermore, the recurrence was limited to the thoracic spine (18).

Our patient had difficulty walking due to the thoracic spine metastasis and compression of the spinal cord. Combination therapy with ZOL and CTX for unresectable rectal cancer with bone metastases was very effective in improving the patient's ability to walk. The compression of the spinal cord diminished after treatment and the patient did not complain of any more difficulty walking while he was alive.

It is not possible to determine whether CTX and ZOL, alone or in combination, had antitumor effects. Their effectiveness against the lung metastasis may be due to the re-administration of CTX. However, as mentioned above, the effectiveness of anti-EGFR therapy for bone metastases is uncertain and the administration of ZOL may have contributed to the reduction in tumor size. These findings suggest that ZOL may play an important role as an effector for cancer therapy. To the best of our knowledge, this is the first report of the clinical course of a patient while under treatment with the combination of ZOL and CTX.
In conclusion, our results demonstrated that combination therapy with ZOL and CTX inhibited the growth of metastatic rectal cancer. Therefore, the efficacy of this combination therapy should be proven in future clinical trials.

\section{Acknowledgements}

Not applicable.

\section{Funding}

No funding was received.

\section{Availability of data and materials}

Not applicable.

\section{Ethics approval and consent to participate}

Not applicable.

\section{Patient consent for publication}

Written informed consent was obtained from the patient for the publication of this case report and accompanying images.

\section{Authors' contributions}

The supervision of the current study was by KY; YT, NM, TaT, ToT, SM, HI, YT, KY interpreted the clinical data and YT and NM wrote, reviewed and/or revised the manuscript.

\section{Competing interests}

The authors declare that they have no competing interests. 


\section{References}

1. Bray F, Ferlay J, Soerjomataram J, Siegel RL, Torre LA and Jemal A: Global cancer statistics, 2018 GLOBOCAN estimates of incidence and mortality worldwide for 36 cancers in 185 countries. CA Cance J Clin 68: 394-424, 2018.

2. Van Cutsem E, Oliveira J and ESMO Guidelines Working Group: Advanced colorectal cancer: ESMO clinical recommendations for diagnosis, treatment and follow-up. Ann Oncol 4 (Suppl): S61-S63, 2009.

3. Goldstein NI, Prewett M, Zuklys K, Rockwell P and Mendelsohn J: Biological efficacy of a chimeric antibody to the epidermal growth factor receptor in a human tumor xenograft model. Clin Cancer Res 1: 1311-1318, 1995.

4. Li S, Schmitz KR, Jeffrey PD, Wiltzius JJ, Kussie P and Ferguson KM: Structural basis for inhibition of the epidermal growth factor receptor by cetuximab. Cancer Cell 7: 301-311, 2005.

5. Bokemeyer C, Bondarenko I, Hartmann JT, de Braud F, Schuch G, Zubel A, Celik I, Schlichting M and Koralewski P: Efficacy according to biomarker status of cetuximab plus FOLFOX-4 as first-line treatment for metastatic colorectal cancer: The OPUS study. Ann Oncol 22: 1535-1546, 2011.

6. Van Cutsem E, Kohne CH, Hitre E, Zaluski J, Chang Chien CR, Makhson A, D'Haens G, Pintér T, Lim R, Bodoky G, et al: Cetuximab and chemotherapy as initial treatment for metastatic colorectal cancer. N Engl J Med 360: 1408-1417, 2009.

7. Corey E, Brown LG, Quinn JE, Poot M, Roudier MP, Higano CS and Vessella RL: Zoledronic acid exhibits inhibitory effects on osteoblastic and osteolytic metastases of prostate cancer. Clin Cancer Res 9: 295-306, 2003.

8. Kato J, Futamura M, Kanematsu M, Gaowa S, Mori R, Tanahashi T, Matsuhashi $\mathrm{N}$ and Yoshida K: Combination therapy with zoledronic acid and cetuximab effectively suppresses growth of colorectal cancer cells regardless of KRAS status. Int J Cancer 138: 1516-1527, 2016.

9. HurwitzH,Fehrenbacher L, Novotny W, Cartwright T, Hainsworth J, Heim W, Berlin J, Baron A, Griffing S, Holmgren E, et al: Bevacizumab plus irinotecan, fluorouracil, and leucovorin for metastatic colorectal cancer. N Engl J Med 350: 2335-2342, 2004.
10. Tournigand C, Andre T, Achille E, Lledo G, Flesh M, Mery-Mignard D, Quinaux E, Couteau C, Buyse M, Ganem G, et al: FOLFIRI followed by FOLFOX6 or the reverse sequence in advanced colorectal cancer: A randomized GERCOR study. J Clin Oncol 22: 229-237, 2004.

11. Cunningham D, Humblet Y, Siena S, Khayat D, Bleiberg H, Santoro A, Bets D, Mueser M, Harstrick A, Verslype C, et al: Cetuximab monotherapy and cetuximab plus irinotecan in irinotecan-refractory metastatic colorectal cancer. N Engl J Med 351: 337-345, 2004.

12. Lewis TS, Shapiro PS and Ahn NG: Signal transduction through MAP kinase cascades. Adv Cancer Res 74: 49-139, 1998.

13. Vivanco I and Sawyers CL: The phosphatidylinositol 3-Kinase AKT pathway in human cancer. Nat Rev Cancer 2: 489-501, 2002.

14. Han FS, Lin MB, Zhu HY, Chen YQ, Shui W and Xu JM: Anti-proliferation effect of zoledronic acid on human colon cancer line SW480. Asian Pac J Trop Med 9: 168-171, 2016.

15. Schubbert S, Shannon K and Bollag G: Hyperactive Ras in developmental disorders and cancer. Nat Rev Cancer 7: 295-308, 2007.

16. Correale P, Marra M, Remondo C, Migali C, Misso G, Arcuri FP, Del Vecchio MT, Carducci A, Loiacono L, Tassone P, et al: Cytotoxic drugs up-regulate epidermal growth factor receptor (EGFR) expression in colon cancer cells and enhance their susceptibility to EGFR-targeted antibody-dependent cell-mediated-cytotoxicity (ADCC). Eur J Cancer 46: 1703-1711, 2010.

17. Maniar A, Zhang X, Lin W, Gastman BR, Pauza CD, Strome SE and Chapoval AI: Human gammadelta T lymphocytes induce robust NK cell-mediated antitumor cytotoxicity through CD137 engagement. Blood 116: 1726-1733, 2010.

18. Nakamura S, Fukui T, Suzuki S, Takeda H, Watanabe K and Yoshioka T: Long-term survival after a favorable response to anti-EGFR antibody plus chemotherapy to treat bone marrow metastasis: A case report of KRAS-wildtype rectal cancer. Onco Targets Ther 10: 1143-1147, 2017.

(i) (8) This work is licensed under a Creative Commons Attribution-NonCommercial-NoDerivatives 4.0 International (CC BY-NC-ND 4.0) License. 